\section{Foliicolous fungi from the Achankovil forests in Kollam District of Kerala State, India}

\author{
V.B. Hosagoudar ${ }^{1}$, P.J. Robin ${ }^{2} \&$ B. Shivaraju ${ }^{3}$ \\ 1,2 Tropical Botanic Garden and Research Institute, Palode, \\ Thiruvananthapuram, Kerala 695562, India \\ ${ }^{3}$ Rehabilitation Planatations Ltd.,Punalur, Kerala 691305, India \\ Email: ${ }^{1}$ vbhosagoudar@rediffmail.com; ${ }^{3}$ mdrpl@sancharnet.in
}

Fungi are an important components of the ecosystem and are to be studied in all aspect because they play a vital role in the forestry and its management. They act as scavengers by degrading litter, break down dead wood as wood rot fungi, infect leaves as parasites, etc. Parasitic fungi may be pathogenic or biotropic. Pathogenic fungi cause diseases by producing symptoms such as leaf spots, hypertrophy, canker, die-back, etc. To understand much about these microbes, their occurrence, interaction with the plants is necessary and urgently needed in all the biodiversity rich forest areas.

Achankovil is a place of pilgrims located in Kollam District of Kerala. The Achankovil forest range, is bounded by Tamil Nadu state in the east, Ranni forest division in the northeast, Konni forest division in the west, Punalur forest division in the southwest and Thenmala forest division in the south. It has an area of $29169 \mathrm{ha}$, comprising dry deciduous, moist deciduous and evergreen forests, with riparian vegetation all along the river Achankovil, which

Date of publication (online): 26 March 2010

Date of publication (print): 26 March 2010

ISSN $0974-7907$ (online) | 0974-7893 (print)

Editor: Vatsavaya S. Raju

Manuscript details:

Ms \# 02323

Received 23 September 2009

Final received 07 December 2009

Finally accepted 08 February 2010

Citation: Hosagoudar, V.B., P.J. Robin \& B. Shivaraju (2010). Foliicolous fungi from the Achankovil forests in Kollam District of Kerala State, India. Journal of Threatened Taxa 2(3): 760-761.

Copyright: (C) V.B. Hosagoudar, P.J. Robin \& B. Shivaraju 2010. Creative Commons Attribution 3.0 Unported License. JoTT allows unrestricted use of this article in any medium for non-profit purposes, reproduction and distribution by providing adequate credit to the authors and the source of publication.

Acknowledgements: We thank Mr. Amarnath Shetty, Chief Conservato of Forests for the facilities provided during the field collection trip and to Shri G. J. Teggi, Chief Conservator of Forests, Kollam, for providing needful information on this forest. We are glad to acknowledge Dr. A. Subramoniam, Director, TBGRI, Palode for providing the facilities.

\section{OPEN ACGESS | FREE DOWNLOAD}

passes through the forest. Teak is extensively planted. A short time visit by trekking from forest office to Amarakkathodu was conducted on 04 December 2008 by the authors to know the preponderance of foliicolous fungi. The present study is the first of its kind in the forest division (Hosagoudar et al. 1996; Sankaran et al. 1997; Florence 2004). The following is the genus and species-wise alphabetical enumeration of foliicolous fungi from Achankovil forests:

\section{Enumeration}

1. Asteridiella combreti (F. Stevens) Hansf. var. leonensis Hansf., Sydowia Beih. 2: 160, 1961; Hosag. \& Goos, Mycotaxon 36: 238, 1989; Hosag., Meliolales of India, p. 83, 1996 (Meliolaceae).

On leaves of Calycopteris florubunda (Roxb.) Lam. (Combretaceae), Amarakkathodu, Achankovil, Kollam, 4.xii.2008, V.B. Hosagoudar et al. HCIO 49025, TBGT 3280.

2. Asterina deightonii Syd., Ann. Mycol. 36: 172, 1938; Hosag. \& Abraham, J. Econ. Taxon. Bot. 4: 571, 2000; Hosag., C.K. Biju, Abraham \& Agarwal, Indian Phytopath. 55: 497, 2002; Hosag., Zoos' Print J. 21: 2326, 2006 (Asterinaceae).

On leaves of Loranthus sp. (Loranthaceae), Amarakkathodu, Achankovil, Kollam, 4.xii.2008, V.B. Hosagoudar et al. HCIO 49021, TBGT 3276.

3. Asterina tertia Racib. in Theissen, Die Gattung Asterina: 103, 1913; Sacc., Sylloge Fungorum 24: 443, 1926; Hosag. \& Abraham, J. Econ. Taxon. Bot. 4: 558, 2000; Hosag., H. Biju \& Appaiah, J. Mycopathol. Res. 43: 204, 2005; 44:12, 2006; 21: 2329, 2006 (Asterinaceae).

On leaves of Acanthaceae member, Amarakkathodu, Achankovil, Kollam, Dec. 4, 2008, V.B. Hosagoudar et al. TBGT 3282; Priya Estate, Achankovil, Kollam, 4.xii.2008, V.B. Hosagoudar et al. HCIO 49028, TBGT 3283.

4. Asterina wrightiae Syd. in Ann. Mycol. 29: 236, 1931; Hosag. \& Abraham, Indian Phytopath. 51: 390, 1998; J. Econ. Taxon. Bot. 4: 560, 2000; Hosag., C.K. Biju \& Abraham, J. Econ. Taxon. Bot. 25: 305, 2001; Hosag., Zoos' Print J. 18: 1280, 2003; 21: 2329, 2006 (Asterinaceae).

On leaves of Wrightia tinctoria (Roxb.) R. Br. (Apocynaceae), Amarakkathodu, Achankovil, Kollam, 4.xii.2008, V.B. Hosagoudar et al. HCIO 49026, TBGT 3281.

5. Asterostomella dilleniacearum Hosag., Abraham \& C.K. Biju, New Botanist 24: 19, 1997; Hosag., C.K. Biju \& Abraham, J. Econ. Taxon. Bot. 25: 307, 2001 (Asterinaceae). 
On leaves of Dillenia pentagyna Roxb. (Dilleniaceae), Amarakkathodu, Achankovil, Kollam, 4.xii.2008, V.B. Hosagoudar et al. HCIO 49023, TBGT 3278.

6. Colemaniella ossoorii Agnihothr., J. Coffee Res. 4: 3 , 1974; Ellis, More Dematiaceous Hyphomycetes, p. 366, 1976; Karandikar \& Patwardhan, Biovigyanam 11: 143, 1985 (Family incertae sedis, Ascomycota).

On leaves of Terminalia sp. (Combretaceae), Koorampala, Achankovil, Kollam, Dec. 4, 2008, V.B. Hosagoudar et al. HCIO 49020, TBGT 3275.

7. Meliola cadigensis H.S. Yates var. glycosmidis (J.N. Kapoor) Hosag., Cryptog. Bot. 2: 186, 1991; Hosag., Meliolales of India, p. 149, 1996. Meliola glycosmidis J.N. Kapoor, Indian Phytopathol. 20: 153, 1967 (Meliolaceae).

On leaves of Glycosmis pentaphylla (Retz.) DC. (Rutaceae), Koorampala, Achankovil, Kollam, 4.xii.2008, V.B. Hosagoudar et al. HCIO 49022, TBGT 3277.

8. Meliola capensis (Kalchbr. \& Cooke) Theiss. var. schleicherae Hosag. \& C.M. Pillai in Hosag., Raghu \& Pillai, Nova Hedwigia 58: 537, 1994; Hosag., Meliolales of India, p. 157, 1996 (Meliolaceae).

On leaves of Schleichera oleosa (Lour.) Oken (Sapindaceae), Amarakkathodu, Achankovil, Kollam, 4.xii.2008, V.B. Hosagoudar et al. HCIO 49030, TBGT 3285.

9. Meliola desmodii-pulchelli Hosag., T.K. Abraham \& C.K. Biju, J. Mycopathol. Res. 37: 94, 1999; Hosag., Meliolales of India 2: 226, 1998 (Meliolaceae).

On leaves of Desmodium pulchellum (L.) Benth. (Papilionaceae), Amarakkathodu, Achankovil, Kollam, 4.xii.2008, V.B. Hosagoudar et al. HCIO 48858, TBGT 3234.

10. Meliola desmodii-velutini Hosag. \& Manojk., Indian Phytopath. 57: 459, 2004; Hosag., Meliolales of India 2: 229, 1998 (Meliolaceae).

On leaves of Desmodium velutinum (Willd.) DC. (Papilionaceae), Amarakkathodu, Achankovil, Kollam, 4.xii.2008, V.B. Hosagoudar et al. HCIO 49180, TBGT 3435.

11. Meliola gamsii Hosag. \& Shiburaj, Nova Hedwigia 74: 411, 2002; Hosag., Meliolales of India 2: 248, 1998 (Meliolaceae).

On leaves of Strychnos nux-vomica L. (Strychnaceae), Amarakkathodu, Achankovil, Kollam, 4.xii.2008, V.B. Hosagoudar et al. HCIO 49179, TBGT 3434.

12. Meliola melanoxylonis Hosag. \& C.M. Pillai in Hosag., Raghu \& Pillai, Nova Hedwigia 58: 540, 1994; Hosag., Meliolales of India, p. 255, 1996 (Meliolaceae).

On leaves of Acacia mangium Willd. (Mimosaceae), Achankovil, Kollam, Dec. 4, 2008, V. B. Hosagoudar \& al. HClO 49029, TBGT 3284; A. melanoxylon R. Br., Achankovil, Kollam, 4.xii.2008, V.B. Hosagoudar et al. $\mathrm{HClO}$
49019, TBGT 3274.

13. Meliola panici Earle, Muhlenbergia 1: 12, 1901; Hansf., Sydowia Beih. 2: 745, 1961; Gupta \& Gupta, Indian Phytopathol. 58:390,1985; Hosag. \& Goos, Mycotaxon 42: 136, 1991; Hosag., Meliolales of India, p. 276, 1996 (Meliolaceae).

On leaves of Grass (Poaceae), Amarakkathodu, Achankovil, Kollam, 4.xii.2008, V.B. Hosagoudar et al. HCIO 49024, TBGT 3279

14. Meliola urariae Hosag., Indian Phytopath. 57: 462, 2004; Meliolales of India 2: 350, 1998 (Meliolaceae).

On leaves of Uraria rufescens (DC.) Schindl. (Papilionaceae), Amarakkathodu, Achankovil, Kollam, 4.xii.2008, V.B. Hosagoudar et al. HCIO 49178, TBGT 3433.

\section{References}

Florence, E.J.M. (2004). Biodiversity Documentation of Kerala part II: Microorganisms (Fungi). Kerala Forest Research Institute, Handbook no.17, 1-293pp.

Hosagoudar, V.B., T.K. Abraham \& P. Pushpangadan (1996). Fungi of Kerala. Tropical Botanic Garden and Research Institute, Palode, Thiruvananthapuram.

Sankaran, K.V., E.J. Florence, M. Balasundaran \& K.I. Nawshad (1997). Fungal Diversity, pp. 140-148. In: Thambi, K., M.M. Nayar \& C.S. Nair (eds.). The Natural Resources of Kerala. World Wide Fund for Nature, Thiruvananthapuram, India.

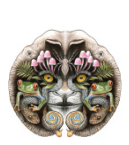

